\title{
Microvascular skin coverage in wounds that expose the Achilles tendon
}

\author{
Baltazar Barrera-Mera ${ }^{1}$, Israel Salgado-Adame ${ }^{2}$, Obed Horacio Grajeda-Chávez ${ }^{3}$, \\ Jazmín Danaé Chávez-Hernández ${ }^{3}$, Cuauhtémoc Aguilar-Barragán', \\ Ana Lilia Abundez-Pliego ${ }^{4}$, Yuri Jiménez-Caprielova ${ }^{5}$, Leonel Dalain Hernández-Arteaga ${ }^{6}$, \\ Román Alberto de la Rosa Llergo ${ }^{7}$, José Enrique Pérez-López ${ }^{8}$, \\ Alan I. Valderrama-Treviño ${ }^{3 *}$
}

\author{
${ }^{1}$ Department of Physiology. Faculty of Medicine. UNAM. CDMX. Mexico \\ ${ }^{2}$ Department of Surgery, Hospital Lic. Adolfo Lopez Mateos, I.S.S.S.T.E., Mexico \\ ${ }^{3}$ Department of Surgery, Central North Hospital PEMEX, CDMX, Mexico \\ ${ }^{4}$ Department of Surgery, PEMEX General Hospital, Ciudad del Carmen, Mexico \\ ${ }^{5}$ Department of Surgery, General Hospital \# 50, I.M.S.S., San Luis Potosi, Mexico \\ ${ }^{6}$ Department of Surgery, General Regional Hospital \#1, I.M.S.S., Morelia, Mexico \\ ${ }^{7}$ Department of Surgery, Hospital Angeles Villahermosa, Mexico \\ ${ }^{8}$ Department of Surgery, High Specialty Regional Hospital Zumpango, Mexico
}

Received: 13 November 2021

Accepted: 17 November 2021

\section{*Correspondence:}

Dr. Alan I. Valderrama-Treviño,

E-mail: alan_valderrama@hotmail.com

Copyright: () the author(s), publisher and licensee Medip Academy. This is an open-access article distributed under the terms of the Creative Commons Attribution Non-Commercial License, which permits unrestricted non-commercial use, distribution, and reproduction in any medium, provided the original work is properly cited.

\begin{abstract}
Achilles tendon rupture is one of the most frequent tendon ruptures. Surgical intervention is the best option, but it has a high rate of complications leading to skin infections and necrosis. Extensive and aggressive debridement is necessary, producing tendon exposure and large skin defects. There are many alternatives for the skin coverage of the Achilles tendon, like sequential closures, vacuum assisted closure, local flaps, regional flaps, and more recently and efficiently microsurgery flaps. Skin defects in the Achilles tendon region have many peculiarities in comparison with other parts of the body: it is a zone that is always under mechanical stress due to footwear, this is why it must be highly resistant but thin. Its coverage must have sensitive properties, endure tendon gliding and allow early rehabilitation. Sometimes tendon reinforcement or creation or a neo tendon will be needed. For fulfilling these requirements free flaps by microsurgery are the only option in $90 \%$ of the cases. In this review article, we analyze three of the most frequently used free flaps in reconstructive medicine for complex Achilles tendon defects; free radial forearm flap, free radial forearm flap with vascularized flexor carpi radialis tendon and free anterolateral thigh (ALT) flap with vascularized fascia lata. This choice will be determined by the number of tissues involved, size of the defect, etiology of the defect, overall condition of the affected extremity and quality of neighboring tissues.
\end{abstract}

Keywords: Achilles tendon surgery, Microsurgery, Free radial forearm flap, Vascularized flexor carpi radialis tendon, Free anterolateral thigh flap, Skin coverage, flap, Compound defect, Composed flaps

\section{INTRODUCTION}

The Achilles tendon is the strongest tendon of the human body it contains several important anatomical entities, such as posterior tibial vessels and tibialis nerve. ${ }^{1-3}$ The soleus and gastrocnemius muscles converge to form the Achilles tendon, which inserts posteriorly on the calcaneus. Its rupture represents one of the most common tendon ruptures, its incidence in the general population is approximately 5 to 10 per $100,000 .{ }^{4}$ There are many 
reasons why it may rupture (Table 1) such as traumatic rupture by direct trauma, diabetes mellitus, drug-induced tendinopathy, fibrosarcoma, secondary to microangiopathy due to peripheral arterial disease, among others.

Table 1: Causes of Achilles tendon exposure.

\begin{tabular}{|l|}
\hline Causes of Achilles tendon exposure \\
\hline Primary to systemic illness \\
\hline Secondary to peripheral arterial disease \\
\hline Direct trauma \\
\hline Iatrogenic complications \\
\hline Following steroid infiltration \\
\hline $\begin{array}{l}\text { Result of surgical repair of ruptures of the } \\
\text { Achilles tendon }\end{array}$ \\
\hline Fibrosarcoma \\
\hline Pyoderma gangrenous \\
\hline
\end{tabular}

Table 2: Complications post-surgical intervention for primary fix.

\begin{tabular}{|l|}
\hline $\begin{array}{l}\text { Complications post-surgical intervention for } \\
\text { primary fix }\end{array}$ \\
\hline Wound dehiscence \\
\hline Infection \\
\hline Skin necrosis \\
\hline Exposure \\
\hline Necrosis of the Achille tendon \\
\hline
\end{tabular}

There is a male to female ratio of $20: 1$ and the average age is $30-50$ years old. ${ }^{5}$ Some of the risk factors that can be related to this rupture are diabetes mellitus, smoking, rheumatoid arthritis in corticosteroid therapy and stress trauma. The treatment of choice is operative management, but there are surgical complications (Table 2) such as secondary rupture, skin necrosis, seroma, fibrotic reaction, wound dehiscence and infection with exposure of tendon. ${ }^{2}$ There is evidence that surgical repair reduced the risk of repeat tendon rupture compared with nonoperative management showing a risk ratio of 0.41 , recurrent rupture after surgery has an incidence of $2-8 \%$ wound infection after surgery has an incidence ranging from $1.7 \%$ to $12.2 \%$, and wound dehiscence happens in $11.8 \%$ of the cases. $^{4-7}$ There is not enough data about amputations due to Achilles tendon surgery failure, but it may be a catastrophic consequence if skin and tendon necrosis is not treated. Whenever these complications happen the management of segmental loss of Achilles tendon injury becomes a complex reconstructive challenge. ${ }^{8}$

Next treatment should be determined by the number of tissues involved, size of the defect, etiology of the defect, overall condition of the affected extremity and quality of neighboring tissues. ${ }^{3}$

The best way to reconstruct a complex soft tissue defect involving the Achilles tendon is addressed here.

\section{METHODS}

A review of available literature was performed using PubMed, ScienceDirect, Scopus and Cochrane databases from 2010 to October 2021. Search criteria were formulated to identify all reports of vascularized, nonvascularized, local and free flaps for Achilles tendon reconstruction in combination with soft tissue coverage. Non-English and animal studies were excluded.

We used the following keywords: Achilles tendon surgery complication, microsurgery, free radial forearm flap, vascularized flexor carpi radialis tendon, free anterolateral thigh flap, skin coverage, flap, Achilles tendon, compound defect, composed flaps.

\section{RESULTS}

When Achilles tendon is widely exposed with associated skin defects, tissue transfer to achieve wound coverages is needed. This may be achieved by using grafts or flaps 2,3 .

Flaps are pieces of tissue that have an integral blood supply, they facilitate tendon healing, prevents and treats infections, as transferred tissue comes with its pedicle, antibiotic acts right in the reconstructed zone. They have also shown a lower rate of tendon adhesion and better gliding capability. ${ }^{2,8}$

Grafts are pieces of tissue detached from its blood supply, biologically and biomechanically not comparable with flaps. These have excellent properties for richly irrigated and healthy zones, useful for small excisional defects around the Achilles region, but they are not a viable option in a wound breakdown when a denuded tendon is exposed. ${ }^{9}$

\section{Reconstruction considerations}

In order to achieve a successful reconstruction, some characteristics must be considered. Reconstruction of the Achilles tendon and skin defects must be done within the first 15 days after the defect was made. Microsurgery represents the cheapest and fastest option, with expected controlled and predictable results. ${ }^{10,11}$ The goals of the treatment should be the creation of durable, soft tissue coverage with sufficient shear strength, approaching preinjury functionality, new richly vascularized zone that responds to neuroendocrine drugs stimuli, resisting shearing forces, endure repetitive friction during walking, achieve an esthetic result, allow smooth tendon gliding and allow normal shoes wearing. ${ }^{2,8}$

\section{Flaps}

Local or regional flaps like propeller flap are usually unable to provide tendon-like structures for the reconstruction of the Achilles tendon, since we are dealing with a small surface area with poor blood circulation, and injuries that caused the defect may have also disrupted the 
vascular supply of the region, harvesting local flaps may endanger the already injured section. ${ }^{12}$

Free flaps, on the other hand, are the best option for combined skin and tendon exposure defects, even though it is more demanding than local regional flaps because it requires microsurgical expertise and longer operative time, it allows simultaneous reconstruction of the Achilles tendon and the overlying soft tissue..$^{12,13}$

Table 3: Advantages and disadvantages of Free flaps.

\begin{tabular}{|c|c|c|}
\hline Free radial forearm flap & $\begin{array}{l}\text { Free radial forearm flap }+ \\
\text { vascularized flexor carpi radialis } \\
\text { tendon }\end{array}$ & $\begin{array}{l}\text { Free anterolateral thigh flap with } \\
\text { vascularized fascia lata }\end{array}$ \\
\hline \multicolumn{3}{|l|}{ Advantages } \\
\hline $\begin{array}{l}\text { For skin defect only } \\
\text { Great for distal anatomical defects } \\
\text { of the leg } \\
\text { Provides gliding vascularized } \\
\text { fascia } \\
\text { Provides thin pliable skin } \\
\text { Provides supple and durable } \\
\text { coverage } \\
\text { Long pedicle length } \\
\text { Large caliber vessels }\end{array}$ & $\begin{array}{l}\text { Great for combined small size } \\
\text { defects } \\
\text { Good functional and aesthetic results } \\
\text { Quality and length of the FCR } \\
\text { tendon } \\
\text { Reliable construction of the tendon } \\
\text { Tendon well supplied by radial artery } \\
\text { Gliding vascularized fascia, thin } \\
\text { pliable skin } \\
\text { Restoration of normal foot function. }\end{array}$ & $\begin{array}{l}\text { Great for combined large size defects } \\
\text { Large dimension } \\
\text { Long reliable pedicle } \\
\text { Adjustable thickness } \\
\text { Rolled fascia lata mimics a tendon }\end{array}$ \\
\hline \multicolumn{3}{|l|}{ Disadvantages } \\
\hline $\begin{array}{l}\text { Donor site morbidity } \\
\text { Donor site needs skin graft } \\
\text { Not suitable for every patient with } \\
\text { comorbidities }\end{array}$ & $\begin{array}{l}\text { Donor site morbidity and uncosmetic } \\
\text { scar in case of skin graft }\end{array}$ & $\begin{array}{l}\text { Rolled fascia does not provide less } \\
\text { strength, gliding ability and insertion } \\
\text { to the calcaneus bone as a real } \\
\text { tendon } \\
\text { Tedious dissection during flap } \\
\text { harvesting }\end{array}$ \\
\hline
\end{tabular}

There are various types such as muscle flap, musculocutaneous flap, fascia lata muscle flap, forearm flaps, among others. Here we are going to analyze the 3 most used free flaps for complex reconstruction of the Achilles tendon. ${ }^{12}$ (Table 3 )

\section{Free radial forearm flap}

Forearm skin flap is vascularized though septocutaneous and musculocuaneous perforators of the radial artery, and microvascular free flaps can be designed around these main arterial systems. ${ }^{14}$ The main feeding vessel is the radial artery, that originates from the brachial artery at around $2 \mathrm{~cm}$ distal of the elbow where the ulnar artery is divided. The radial artery length is about $18 \mathrm{~cm}$ and width of the lumen $3 \mathrm{~mm}$, that allows to perform micro anastomosis. Cephalic vein is the most commonly used single vein for venous drainage, its width is $3 \mathrm{~cm}$ or more. ${ }^{15}$ It is important to confirm hand vascularity with two Allen test or angiography to check the recovery of superficial palmar arch by the ulnar artery. ${ }^{16}$

Helpful when the reconstruction of the Achilles tendon can be done by closing the gap with synthetic or autologous material or transferring muscles like peroneus brevis or flexor digitorum longus. ${ }^{13}$ It is considered as one of the first choices in case of reconstruction of soft-tissue defects located in the distal anatomical districts of the leg 3 , provides gliding vascularized fascia and thin pliable skin of approximately $8 \mathrm{~mm}$ width, provides supple and durable coverage and allows two teams or surgeons to operate simultaneously, decreasing the operation time. Thin flaps have the advantage of allowing the patient normal footwear and neurosensitive reconstruction with brachial lateral neve. The long pedicle length allows for vessel repair outside the zone of injury. The disadvantages that this technique has are esthetic issues and donor site morbidity, necessitating local rotation and advancement flap or skin grafting. Primary closure is only possible with a cutaneous flap of less than $3 \mathrm{~cm} .^{15}$

\section{Composite flaps}

When we must deal with a simultaneous reconstruction of skin and tendon a composite free flap is the safer option. In case of full-thickness necrosis of the tendon this technique combines simultaneous transfer of vascularized fascia and skin or tendon and skin. It is characterized by high resistance to infection, rapid healing, reduced scarring, and improved tendon morbidity. ${ }^{16}$ Fasciocutaneous flaps have shown to have the advantage of providing thin and durable coverage. ${ }^{9}$ Composed flaps can be done in a single step approach, that provides fewer procedures, resulting in quicker overall recovery and decreased surgical risk to the patient compared to multiple- 
step operations that increases the risk of complications and are time and cost consuming. ${ }^{13,17}$

\section{Free radial forearm flap with vascularized flexor carpi radialis tendon}

This technique represents an important option when we are talking about a complex defect of skin and tendon that is small to medium size. ${ }^{18}$ Sensory flap can be achieved by including the anterior branch of the lateral cutaneous nerve. ${ }^{19}$ The advantages of this flaps are the quality of the tendon, which is well supplied by the radial artery, provides the gliding vascularized fascia and thin pliable skin needed for the purpose, the vessels are of large caliber and the long pedicle length allows for vessel repair outside the zone of injury, allows the restoration of normal foot function, permits normal shoe wear and ambulation. ${ }^{3,9}$ The flexor carpi radialis tendon optimally replaces the Achilles tendon, its mechanical strength and the ability to glide in its physiological sheet seem to give this flap advantages over other options. ${ }^{18}$ On the other hand, some of the disadvantages are the visible scars at the donor site, the sacrifice of the radial artery, morbidity in the donor site, and un-cosmetic scar in case of skin graft. ${ }^{3,18}$

\section{Free anterolateral thigh (ALT) flap with vascularized fascia lata}

ALT flap has been used since 1984 for the reconstruction of soft tissue defects all over the body, it is a musculofasciocutaneous flap, harvested as a composite flap with the fascia lata. ${ }^{8}$ Fascia lata is well vascularized and receives its blood supply from its subfascial and perifascial vascular plexus when attaches to the ALT flap. ${ }^{2}$ The descending branch of the lateral circumflex femoral artery supplies the flap in most patients, the artery and two associated concomitant veins transverse obliquely with the nerve. ${ }^{20}$ It can be rolled and mimic a tendon to replace the Achilles tendon defect. ${ }^{12}$ This is a reliable option when we are dealing with a composite large size defect of skin and tendon, and one of the most popular options within trained microsurgeons. ${ }^{12}$ Some of the advantages are that it has a large dimension, has a long reliable pedicle, adjustable thickness, minimal donor site mobility, durable skin quality and it has shown that dorsiflexion and plantar flexion almost fully recovers after reconstruction. ${ }^{3,12}$ On the other hand, the reconstruction of the Achilles tendon with a tubular fascia lata flap is unlikely to provide adequate strength and gliding ability as good as the one achieved by a true tendon-like in flexor carpi radialis tendon, harvestings of the tensor fascia lata can increase donor site morbidity and in some patient's adipose panicle may be too thick, hindering the use of normal footwear. ${ }^{18}$

\section{DISCUSSION}

Achilles tendon is the strongest tendon in the human body 1 and paradoxically it is also the one that ruptures the most. This may be due to the increased risk factors in the society, such as smoking, vascular or metabolic diseases as direct contact injuries. When an Achilles tendon rupture happens the first therapeutic option is surgical management, it can be open or percutaneous. ${ }^{1}$ Open surgery has a woundrelated complication rate between $8.2-34.1 \%$ of which at least half are due to infection because of the zone's poor blood supply. Aggressive debridement is recommended in the case of necrosis and infected tissue, this is mandatory to reduce bacterial account and optimize blood flow of the region leading to wide defects in skin and tendon gaps. ${ }^{18,19}$ This is now considered a reconstructive challenge, owing to the lack of suitable tissue on site 8 and poor results with traditional therapies like vacuum-assisted closure. ${ }^{20}$

Reconstructive medicine is a relatively new but extremely efficient therapy. Grafts are pieces of tissue that are detached from its site and its blood supply, this graft cannot survive on poorly vascularized, densely scared, or infected tissue so it is not a suitable option in this case. ${ }^{21}$ Flaps are pieces of tissue that have an integral blood supply, having higher resistance to infection, faster healing, and fewer adhesions. ${ }^{21}$ Local or regional flaps as propeller flaps are areas of tissue transferred to an adjacent recipient defect, useful for small excisional defects but not a viable option when the tendon is exposed. ${ }^{22}$

Microsurgery has revolutionized reconstructive surgery introducing Free flaps. This technique allows a piece of tissue to be taken from a donor site, including the artery and vein which supply it. ${ }^{21}$ Free radial forearm flap is extremely useful when there is an important skin defect but the tendon may be fixed using other methods, as Nazerali et al did by reconstructing the Achilles tendon with the flexor hallucis longus that is the second stronger tendon in the region, they used free radial forearm flap, and showed to provide good restoration of plantar flexion and stable coverage over the Achilles tendon, allowing normal footwear. ${ }^{13}$ When there is a skin and tendon defect it is called composite defects, for these, the best option is to reconstruct with a composite free flap of skin and tendon or skin and fascia that can be rolled up and used as a tendon. ${ }^{18}$ Free radial forearm flap with vascularized flexor carpi radialis (FCR) tendon, is a composed flap for small or medium composite defects, its tensile strength resists the secondary breakdown of the reconstructed tendon, promotes control over the local infection, provides protective sensation for the prevention of skin breakdown and supplement ankle motion. ${ }^{23}$ It provides good aesthetic results with few disadvantages Table 3 . Innocenti et al. made a free radial forearm flap with vascularized flexor carpi radialis tendon for 6 patients who had a post-surgical infection in the Achilles tendon, as results they saw that all the flaps survived and no complications were recorded, optimal reconstruction of the tendon and range motion was minimally reduced if compared to the contralateral side. ${ }^{18}$ Free anterolateral thigh (ALT) flap with vascularized fascia lata is one of the favorite methods used by microvascular surgeons, it's an excellent choice for large size defects, the harvested flap can have any desirable size and the wide vessels and long pedicle facilitates vascular reconnection, but it requires specialized equipment and 
personal and it's an elevate technique as fascia has to be dissected and then rolled into a tendon shape. ${ }^{24}$ Some authors believe that despite its advantage's fascia will never have the same properties as a real tendon. Ehrl et al used this flap in patients with complex Achilles tendon defects and had a reliable and safe reconstruction, achieving functional results comparable to the unaffected side. ${ }^{19,22}$

\section{CONCLUSION}

The ideal procedure for a complex Achilles tendon defect depends on the patients' characteristics. In younger patients, a full recovery range of motion is desired as well as a strong tendon that will endure friction forces. If we are talking about a tendon rupture that can be fixed with suture but has an important skin defect the free radial forearm flap is a suitable option. Talking about a complex defect of tendon and skin, a composed flap is the better option as it deals simultaneously with both issues. Deciding between free radial forearm flap with vascularized flexor carpi radialis tendon and free anterolateral thigh flap with vascularized fascia lata, which are two of the most employed composed flaps, will be a matter of the defect size. Free radial forearm flap with vascularized flexor carpi radialis tendon is great for combined small to medium size defects, whereas free anterolateral thigh flap with vascularized fascia lata has shown better results for combined large size defects.

Funding: No funding sources Conflict of interest: None declared

Ethical approval: Not required

\section{REFERENCES}

1. Yang X, Meng H, Quan Q, Peng J, Lu S, Wang A. Management of acute Achilles tendon ruptures: a review. None Joint Res. 2018;12:561-9.

2. Jandali Z, Lam M, Merwart B, Mohring B, Geil S, Muller K, et al. Predictors of clinical outcome after reconstruction of complex soft tissue defects involving the Achilles tendon with the composite anterolateral thigh flap with vascularized fascia lata. J Reconstr Microsurg. 2018;2:632-4.

3. Innocenti M, Innocenti A, Ghezzi S, Delcroix L. Achilles region soft tissue defects: a reconstructive algorithm based on a series of 46 cases. J Reconstr Microsurg. 2017;3:40-7.

4. Huttunen TT, Kannus P, Rolf C, Tsai L, Mattila V. Acute achilles tendon ruptures: incidence of injury and surgery in Sweden between 2001 and 2012. Am J Sports Med. 2014;10:2419-23.

5. Cetti R, Christensen SE, Ejsted R, Jensen NM, Jorgensen U. Operative versus nonoperative treatment of Achilles tendon rupture. A prospective randomized study and review of the literature. Am J Sports Med. 1993;4:791-9.

6. Deng S, Sun Z, Zhang C, Chen G, Li J. Surgical treatment versus conservative management for acute
Achilles tendon rupture: a systematic review and meta-analysis of randomized controlled trials. J Foot anke surg. 2017;8:1236-43.

7. Ozkaya U, Parmaksizoglu AS, Kabukcuoglu Y, Sokucu S, Basilgan S. Open minimally invasive Achilles tendon repair with early rehabilitation: functional results of 25 consecutive patients. Injury. 2009;7:669-72.

8. Houtmeyers P, Opsomer D, Van Landuyt K, Monstrey S. Reconstruction of the Achilles tendon and overlying soft tissue by free composite anterolateral thigh flap with vascularized fascia lata. J Reconstr Microsurg. 2012;7:205-9.

9. Chun K, Margoles S, Birnbaum J. Radial forearm free flap for salvage of Achilles-tendon repair wounds. J Reconstr Microsurg. 2000;519-23.

10. Banegas-Ruiz R, Valderrama-Treviño AI, RivasMontero JA, Gómez-Mendoza FF, García-Martínez E, Domínguez BCR, et al. Antebrachial microvascular flap for reconstruction of the pelvic limb: case report. Int J Res Med Sci. 2019;8:1952-6.

11. Banegas-Ruiz R, Gómez-Mendoza FF, BacaDomínguez CR, Campos-Angulo G, Alejo-Fuentes LJ, Alejo-Fuentes LF, et al. Reverse-flow anterolateral thigh flap for knee soft-tissue reconstruction: case report. Int J Res Med Sci. 2019;9:3914-6.

12. Ki S, Wha S, Hwan Y, Tae K. The composite anterolateral thigh flap for Achilles tendon and soft tissue defect reconstruction with tendon repair by fascia with double or triple folding technique. Microsurgery. 2015;10:615-21.

13. Nazerali R, Hakimi M, Giza E, Sahar D. Single-stage reconstruction of Achilles tendon rupture with flexor hallucis longus tendon transfer and simultaneous free radial fasciocutaneous forearm flap. Ann Plast Surg. 2013;10:416-8.

14. Acarturk TO, Tuncer U, Aydogan LB, Dalay AC. Median artery arising from the radial artery: its significance during harvest of a radial forearm free flap. J Plast Reconstr Aesthet Surg. 2008;11:5-8.

15. Hoo Y, Nam W, Cha I, Jun H. Revisiting radial forearm free flap for successful venous drainage. Maxillofacial Plastic and reconstructive surgery. 2017;1:14.

16. Marchesi A, Parodi P, Brioschi M, Riccio M, Perrotta E, Colombo M, et al. Soft-tissue defects of the Achilles tendon region: Management and reconstructive Ladder. Review of the Literature. Injury. 2016;3:47:S14753.

17. Sato R, Hibino N, Yamano M, Yoshioka S, Terai T, Kenichiro K, et al. One stage reconstruction for re rupture of Achilles Tendon with soft tissue infections: using an anterolateral thigh flap incorporating a vascularized muscle flap and a strip of iliotibial tract. J Hand Microsurg. 2018;2:105-8.

18. Innocenti M, Tani M, Carulli C, Ghezzi S, Raspanti A, Menichini G. Radial forearm flap plus flexor carpi radialis tendon in Achilles Tendon reconstruction: 
Surgical Technique, functional results, and gait analysis. Microsurgery. 2015;9:608-14.

19. DeFazio M, Han K, Lorio M, Attinger C, Steinberg $\mathrm{J}$, Kim P, et al. Combined free tissue transfer for the Management of Composite Achilles defects: functional outcomes and patient satisfaction following thigh-based vascularized reconstruction with a neo tendon construct. J Reconstr Microsug. 2014;4:431-40.

20. Soons J, Rakhorst H, Ruettermann M, Luijsterburg A, Bos P, Zophel O. Reconstruction of defects involving the Achilles Tendon and local Soft Tissues: A quick solution for a Lingering problem. Bone Joint J. 2015;2:215-20.

21. Dun R. Grafts and Flaps. Surgery. 2006;27-32.

22. Jakubietsz R, Jakubietz D, Gruenert J, Schmidit R, Meffert R, Jakubietz M. Reconstruction of soft tissue defects of the Achilles tendon with rotation flaps, pedicled propeller flaps and free perforator flaps. Microsurgery. 2010;2:608-13.

23. Cheol-Hann K, Min-Seong T, Chang-Yong C, SangGue K, Yong-Bae K. A single-stage reconstruction of a complex Achilles wound with modified free composite lateral arm flap. J Reconstr microsurg. 2008;1:127-30.

24. Ehrl D, Heidekrueger P, Schmitt A, Liska F, Ninkovic M, Giunta R, et al. The anterolateral thigh flap for Achilles Tendon reconstruction: Functional Outcomes. Plast Reconstr Surg. 2019;11:1772-83.

Cite this article as: Barrera-Mera B, SalgadoAdame I, Grajeda-Chávez OH, Chávez-Hernández JD, Aguilar-Barragán C, Abundez-Pliego AL et al. Microvascular skin coverage in wounds that expose the Achilles tendon. Int J Res Med Sci 2021;9:37338. 